\title{
Article
}

\section{A variety of uncertainty principles for the Hankel-Stockwell transform}

\author{
Khaled Hleili \\ Preparatory Institute for Engineering Studies of Kairouan, Department of Mathematics, Kairouan university, Tunisia. \\ Department of Mathematics, Faculty of Science, Northern Borders University, Arar, Saudi Arabia.; \\ khaled.hleili@gmail.com \\ Academic Editor: Irshaad Ahmed \\ Received: 24 December 2020; Accepted: 21 January 2021; Published: 29 January 2021.
}

\begin{abstract}
In this work, we establish $L^{p}$ local uncertainty principle for the Hankel-Stockwell transform and we deduce $L^{p}$ version of Heisenberg-Pauli-Weyl uncertainty principle. Next, By combining these principles and the techniques of Donoho-Stark we present uncertainty principles of concentration type in the $L^{p}$ theory, when $1<p \leqslant 2$. Finally, Pitt's inequality and Beckner's uncertainty principle are proved for this transform.
\end{abstract}

Keywords: Hankel-Stockwell transform, local uncertainty principles, Heisenberg-Pauli-Weyl inequality, concentration uncertainty principles, Pitt's inequality, Beckner's inequality.

MSC: 42B10, 42C40.

\section{Introduction}

I $\mathrm{n}$ harmonic analysis, uncertainty principles play an important role. It states that a non-zero function and its Fourier transform cannot be simultaneously sharply concentrated. many of them have already been studied from several points of view for the Fourier transform, Heisenberg-Pauli-Weyl inequality [1] and local uncertainty inequality [2]. As a classical uncertainty principle, the Heisenberg uncertainty principle has been extended to transforms such as the spherical mean transforms [3,4], the Dunkl transform [5] and so forth.

The Hankel transform $\mathcal{H}_{\alpha}$ is defined for every integrable function $f$ on $\mathbb{R}_{+}=[0,+\infty[$ with respect to the measure $d v_{\alpha}$, by

$$
\mathcal{H}_{\alpha}(f)(\lambda)=\int_{0}^{+\infty} f(x) j_{\alpha}(\lambda x) d v_{\alpha}(x)
$$

where $d v_{\alpha}$ is the measure defined on $\mathbb{R}_{+}$by

$$
d v_{\alpha}(x)=\frac{x^{2 \alpha+1}}{2^{\alpha} \Gamma(\alpha+1)} d x
$$

and $j_{\alpha}$ is the modified Bessel function given in the next section.

The Hankel transform is found as a very useful mathematical tool in many fields of physics, signal processing and other [6,7]. Also, many uncertainty principles related to this transform $\mathcal{H}_{\alpha}$ have been proved [8-10].

Time-frequency analysis plays an important role in harmonic analysis, in particular in signal theory. With the development of time-frequency analysis, the study of uncertainty principles have gained considerable attention and have been extended to a wide class of integral transforms such as Weinstein transforms [11,12], Dunkl transforms [13], Hankel-Stockwell transforms [14] and so on.

Based on the ideas of Faris [15] and Price [2,16], we show a general form of the local uncertainty principles for the Hankel-Stockwell transform and we deduce $L^{p}$ version of Heisenberg-Pauli-Weyl uncertainty principle. We shall use also the Heisenberg uncertainty principle, the properties of the Hankel-Stockwell transform and the techniques of Donoho-Stark $[17,18]$, we show a continuous-time principle for the $L^{p}$ theory, when $1<p \leqslant$ 2. Finally, Pitt's inequality and Beckner's uncertainty principle are proved for this transform. 
This work is organized as follows; in Section 2 we recall some harmonic analysis results related to the Hankel transform. In Section 3, we present some elements of harmonic analysis related to the Hankel-Stockwell transform. In Section 4, we introduce some uncertainty principles for this transform.

\section{The Hankel transform}

In this section, we summarize some harmonic analysis tools related to the Hankel transform that will be used hereafter, (see [19]). The modified Bessel function $x \longmapsto j_{\alpha}(x)$ has the following integral representation [20,21];

$$
j_{\alpha}(x)= \begin{cases}\frac{2 \Gamma(\alpha+1)}{\sqrt{\pi} \Gamma\left(\alpha+\frac{1}{2}\right)} \int_{0}^{1}\left(1-t^{2}\right)^{\alpha-\frac{1}{2}} \cos (x t) d t, & \text { if } \alpha>\frac{-1}{2} \\ \cos x, & \text { if } \alpha=\frac{-1}{2} .\end{cases}
$$

In particular, for every $x \in \mathbb{R}$ and $k \in \mathbb{N}$, we have

$$
\left|j_{\alpha}^{(k)}(x)\right| \leqslant 1
$$

We define the Hankel translation operators $\tau_{x}, x \in[0,+\infty[$ by

$$
\tau_{x}(f)(y)= \begin{cases}\frac{\Gamma(\alpha+1)}{\sqrt{\pi} \Gamma\left(\alpha+\frac{1}{2}\right)} \int_{0}^{\pi} f\left(\sqrt{x^{2}+y^{2}+2 x y \cos \theta}, x+y\right) \sin ^{2 \alpha}(\theta) d \theta, & \text { if } \alpha>\frac{-1}{2} \\ \frac{f(x+y)+f(|x-y|)}{2}, & \text { if } \alpha=\frac{-1}{2}\end{cases}
$$

whenever the integral in the right-hand side is well defined. In the following, we denote by;

- $S_{e}(\mathbb{R})$ the Schwartz space, constituted by the even infinitely differentiable functions on the real line, rapidly decreasing together with all their derivatives,

- $L^{p}\left(d v_{\alpha}\right)$ the Lebesgue space of measurable functions $f$ on $\mathbb{R}_{+}$, such that $\|f\|_{p, v_{\alpha}}<+\infty$.

For every $f \in L^{p}\left(d v_{\alpha}\right), p \in[1,+\infty]$, and for every $x \in \mathbb{R}_{+}$, the function $\tau_{x}(f)$ belongs to the space $L^{p}\left(d v_{\alpha}\right)$ and

$$
\left\|\tau_{x}(f)\right\|_{p, v_{\alpha}} \leqslant\|f\|_{p, v_{\alpha}}
$$

In particular, for every $x, y \in \mathbb{R}_{+}$, we have

$$
\tau_{x}(f)(y)=\tau_{y}(f)(x)
$$

If $f \in L^{1}\left(d v_{\alpha}\right)$, then

$$
\int_{0}^{+\infty} \tau_{x}(f)(y) d v_{\alpha}(y)=\int_{0}^{+\infty} f(y) d v_{\alpha}(y) .
$$

The convolution product of $f, g \in L^{1}\left(d v_{\alpha}\right)$ is defined by

$$
f * g(x)=\int_{0}^{+\infty} \tau_{x}(f)(y) g(y) d v_{\alpha}(y)
$$

Let $p, q, r \in[1,+\infty]$ such that $\frac{1}{p}+\frac{1}{q}=1+\frac{1}{r}$. Then for every $f \in L^{p}\left(d v_{\alpha}\right)$ and $g \in L^{q}\left(d v_{\alpha}\right)$, the function $f * g$ belongs to the space $L^{r}\left(d v_{\alpha}\right)$, and we have the following Young's inequality

$$
\|f * g\|_{r, v_{\alpha}} \leqslant\|f\|_{p, v_{\alpha}}\|g\|_{q, v_{\alpha}} .
$$

The Hankel transform $\mathcal{H}_{\alpha}$ is defined on $L^{1}\left(d v_{\alpha}\right)$ by

$$
\forall \lambda \in \mathbb{R} ; \mathcal{H}_{\alpha}(f)(\lambda)=\int_{0}^{+\infty} f(x) j_{\alpha}(\lambda x) d v_{\alpha}(x) .
$$


Theorem 1. (1) [Inversion formula] Let $f \in L^{1}\left(d v_{\alpha}\right)$ such that $\mathcal{H}_{\alpha}(f) \in L^{1}\left(d v_{\alpha}\right)$, then we have

$$
f(x)=\int_{0}^{+\infty} \mathcal{H}_{\alpha}(f)(\lambda) j_{\alpha}(\lambda x) d v_{\alpha}(\lambda) \text {, a.e. }
$$

(2) [Plancherel theorem] The Fourier transform $\mathcal{H}_{\alpha}$ can be extended to an isometric isomorphism from $L^{2}\left(d v_{\alpha}\right)$ onto itself and we have

$$
\left\|\mathcal{H}_{\alpha}(f)\right\|_{2, v_{\alpha}}=\|f\|_{2, v_{\alpha}} .
$$

(3) [Parseval's formula] For all functions $f$ and $g$ in $L^{2}\left(d v_{\alpha}\right)$, we have

$$
\int_{0}^{+\infty} f(x) \overline{g(x)} d v_{\alpha}(x)=\int_{0}^{+\infty} \mathcal{H}_{\alpha}(f)(\lambda) \overline{\mathcal{H}_{\alpha}(g)(\lambda)} d v_{\alpha}(\lambda) .
$$

The Hankel transform $\mathcal{H}_{\alpha}$ satisfies the following properties;

For every $f \in L^{1}\left(d v_{\alpha}\right)$ and $g \in L^{p}\left(d v_{\alpha}\right), p=1,2$, the function $f * g$ belongs to $L^{p}\left(d v_{\alpha}\right), p=1,2$, and we have

$$
\mathcal{H}_{\alpha}(f * g)=\mathcal{H}_{\alpha}(f) \mathcal{H}_{\alpha}(g) .
$$

Let $f, g \in L^{2}\left(d v_{\alpha}\right)$. Then $f * g \in L^{2}\left(d v_{\alpha}\right)$, if and only if $\mathcal{H}_{\alpha}(f) \mathcal{H}_{\alpha}(g) \in L^{2}\left(d v_{\alpha}\right)$ and we have

$$
\mathcal{H}_{\alpha}(f * g)=\mathcal{H}_{\alpha}(f) \mathcal{H}_{\alpha}(g),
$$

moreover,

$$
\int_{0}^{+\infty}|f * g(x)|^{2} d v_{\alpha}(x)=\int_{0}^{+\infty}\left|\mathcal{H}_{\alpha}(f)(\lambda)\right|^{2}\left|\mathcal{H}_{\alpha}(g)(\lambda)\right|^{2} d v_{\alpha}(\lambda),
$$

where both integrals are finite or infinite.

\section{Hankel-Stockwell transform}

We recall some results introduced and proved in [14]. The modulation operator is defined for every function $\psi$ in $L^{2}\left(d v_{\alpha}\right)$ by

$$
M_{a}(\psi)=\mathcal{H}_{\alpha}\left(\sqrt{\tau_{a}\left(\left|\mathcal{H}_{\alpha}(\psi)\right|^{2}\right)}\right), \quad a>0 .
$$

Then for every $\psi \in L^{2}\left(d v_{\alpha}\right), M_{a}(\psi)$ belongs to $L^{2}\left(d v_{\alpha}\right)$ and we have

$$
\left\|M_{a}(\psi)\right\|_{2, v_{\alpha}}=\|\psi\|_{2, v_{\alpha}} .
$$

Now, for every $\psi \in L^{2}\left(d v_{\alpha}\right)$, we consider the family $\psi^{a, r},(a, r) \in \mathbb{R}_{+}^{*} \times \mathbb{R}_{+}$defined by

$$
\forall x \in \mathbb{R}_{+}, \quad \psi^{a, r}(x)=\tau_{r} M_{a} D_{a} \psi(x),
$$

where $D_{a}$ is the dilatation operator given by

$$
D_{a}(\psi)(x)=a^{\alpha+1} \psi(a x) .
$$

Then, we have the following properties;

(i) For every $\psi \in L^{2}\left(d v_{\alpha}\right)$

$$
\tau_{x} D_{a}(\psi)=D_{a} \tau_{a} x(\psi)
$$

(ii) For every $\psi \in L^{2}\left(d v_{\alpha}\right)$

$$
\mathcal{H}_{\alpha}\left(D_{a}(\psi)\right)=D_{\frac{1}{a}}\left(\mathcal{H}_{\alpha}(\psi)\right) .
$$

Definition 1. A nonzero function $\psi \in L^{2}\left(d v_{\alpha}\right)$ is said to be an admissible window function if

$$
0<C_{\psi}=\frac{1}{2^{\alpha} \Gamma(\alpha+1)} \int_{0}^{+\infty} \tau_{1}\left(\left|\mathcal{H}_{\alpha}(\psi)\right|^{2}\right)(a) \frac{d a}{a}<+\infty .
$$


In the following we denote by $\mu_{\alpha}$ the measure defined on $\mathbb{R}_{+}^{*} \times \mathbb{R}_{+}$by

$$
d \mu_{\alpha}(a, r)=d v_{\alpha}(a) d v_{\alpha}(r)
$$

and $L^{p}\left(d \mu_{\alpha}\right), 1 \leqslant p \leqslant+\infty$, the Lebesgue space on $\mathbb{R}_{+}^{*} \times \mathbb{R}_{+}$with respect to the measure $\mu_{\alpha}$ with the $L^{p}$-norm denoted by $\|\cdot\|_{p, \mu_{\alpha}}$.

Definition 2. Let $\psi$ be an admissible window function. The continuous Hankel-Stockwell transform $S_{\psi}^{\alpha}$ is defined in $L^{2}\left(d v_{\alpha}\right)$ by

$$
\begin{aligned}
S_{\psi}^{\alpha}(f)(a, r) & =\int_{0}^{+\infty} f(s) \overline{\psi^{a, r}(s)} d v_{\alpha}(s) \\
& =f * M_{a} D_{a}(\bar{\psi})(r)=f * D_{a} M_{1}(\bar{\psi})(r)=\left\langle f, \psi^{a, r}\right\rangle_{v_{\alpha}}
\end{aligned}
$$

where $\langle,\rangle_{v_{\alpha}}$ is the usual inner product in the Hilbert space $L^{2}\left(d v_{\alpha}\right)$.

Proposition 1. Let $\psi$ be an admissible window function. For every $f \in L^{2}\left(d v_{\alpha}\right)$, we have

$$
\left\|S_{\psi}^{\alpha}(f)\right\|_{\infty, \mu_{\alpha}} \leqslant\|f\|_{2, v_{\alpha}}\|\psi\|_{2, v_{\alpha}} .
$$

Proposition 2. Let $\psi$ be an admissible window function.

(i) [Plancherel formula] For every $f \in L^{2}\left(d v_{\alpha}\right)$, we have

$$
\left\|S_{\psi}^{\alpha}(f)\right\|_{2, \mu_{\alpha}}=\sqrt{C_{\psi}}\|f\|_{2, \nu_{\alpha}}
$$

(ii) [Parseval formula] For all $f, h \in L^{2}\left(d v_{\alpha}\right)$, we have

$$
\int_{0}^{+\infty} \int_{0}^{+\infty} S_{\psi}^{\alpha}(f)(a, r) \overline{S_{\psi}^{\alpha}(h)(a, r)} d \mu_{\alpha}(a, r)=C_{\psi} \int_{0}^{+\infty} f(s) \overline{h(s)} d v_{\alpha}(s) .
$$

(iii) [Inversion formula] For all $f \in L^{1}\left(d v_{\alpha}\right) \cap L^{2}\left(d v_{\alpha}\right)$, such that $\mathcal{H}_{\alpha}(f)$ belongs to $L^{1}\left(d v_{\alpha}\right)$, we have

$$
f(u)=\frac{1}{C_{\psi}} \int_{0}^{+\infty}\left(\int_{0}^{+\infty} S_{\psi}^{\alpha}(f)(a, r) \psi^{a, r}(u) d v_{\alpha}(r)\right) d v_{\alpha}(a), \text { a.e., }
$$

where for each $u \in \mathbb{R}_{+}$, both the inner integral and the outer integral are absolutely convergent, but possible not the double integral.

By Riesz-Thorin's interpolation theorem we obtain the following.

Proposition 3. Let $\psi$ be an admissible window function, $f \in L^{2}\left(d v_{\alpha}\right)$ and $2 \leqslant p \leqslant+\infty$, then we have

$$
\left\|S_{\psi}^{\alpha}(f)\right\|_{p, \mu_{\alpha}} \leqslant C_{\psi}^{\frac{1}{p}}\|\psi\|_{2, v_{\alpha}}^{1-\frac{2}{p}}\|f\|_{2, v_{\alpha}}
$$

\section{Uncertainty principle for the Hankel-Stockwell transform}

In this section, we obtain some uncertainty principles for the Hankel-Stockwell transform.

Theorem 2. [ $L^{p}$ local uncertainty principle for $S_{\psi}^{\alpha}$ ] Let $\psi$ be an admissible window function and $\Sigma$ be measurable subset of $\mathbb{R}_{+}^{*} \times \mathbb{R}_{+}$such that $0<\mu_{\alpha}(\Sigma)<+\infty$. Let $\left.\left.p \in\right] 1,2\right], q=\frac{p}{p-1}$, then or every $f \in L^{p}\left(d v_{\alpha}\right)$, we have 


$$
\left\|\chi_{\Sigma} S_{\psi}^{\alpha}(f)\right\|_{q, \mu_{\alpha}} \leqslant \begin{cases}C_{1}(b, \psi)\left(\mu_{\alpha}(\Sigma)\right)^{\frac{b}{\alpha+1}}\left(\left\|r^{b} f\right\|_{2 p, v_{\alpha}}+\|\psi\|_{2, v_{\alpha}}^{-\frac{2}{q}}\left\|r^{b} f\right\|_{2, v_{\alpha}}\right), & \text { if } 0<b<\frac{\alpha+1}{q} \\ C_{2}(b, \psi)\left(\mu_{\alpha}(\Sigma)\right)^{\frac{1}{q}}\|f\|_{2 p, v_{\alpha}}^{1-\frac{\alpha+1}{q b}}\left\|r^{b} f\right\|_{2 p, v_{\nu^{\prime}}}^{\frac{\alpha+1}{q b}} & \text { if } b>\frac{\alpha+1}{q} \\ C_{3}(b, \psi)\left(\mu_{\alpha}(\Sigma)\right)^{\frac{1}{2 q}}\left(\|\psi\|_{2, v_{\alpha}}^{-\frac{2}{q}}\|f\|_{2, v_{\alpha}}^{\frac{1}{2}}\left\|r^{b} f\right\|_{2, v_{\alpha}}^{\frac{1}{2}}+\|f\|_{2 p, v_{\alpha}}^{\frac{1}{2}}\left\|r^{b} f\right\|_{2 p, v_{\alpha}}^{\frac{1}{2}}\right), & \text { if } b=\frac{\alpha+1}{q} .\end{cases}
$$

where

$$
\begin{aligned}
& C_{1}(b, \psi)=\left(\frac{1}{2^{\alpha+1} \Gamma(\alpha+1)(\alpha+1-b q)}\right)^{\frac{b}{2(\alpha+1)}} C_{\psi}^{\frac{1}{q}-\frac{b}{\alpha+1}}\|\psi\|_{2, v_{\alpha}}, \\
& C_{2}(b, \psi)=\left(\frac{\Gamma\left(\frac{\alpha+1}{b p}\right) \Gamma\left(\frac{q b-(\alpha+1)}{b p}\right)}{b p 2^{\alpha+1} \Gamma(\alpha+1) \Gamma\left(\frac{q}{p}\right)}\right)^{\frac{1}{2 q}}\left(\frac{q b}{q b-(\alpha+1)}\right)^{\frac{1}{2 p}}\left(\frac{q b}{\alpha+1}-1\right)^{\frac{\alpha+1}{2 q b p}}\|\psi\|_{2, v_{\alpha}}, \text { and } \\
& C_{3}(b, \psi)=2 C_{1}\left(\frac{b}{2}, \psi\right) .
\end{aligned}
$$

Proof. (i) It is clear that the first inequality holds if

$$
\left\|r^{b} f\right\|_{2 p, v_{\alpha}}+\|\psi\|_{2, v_{\alpha}}^{-\frac{2}{\bar{q}}}\left\|r^{b} f\right\|_{2, v_{\alpha}}=+\infty .
$$

Let $f \in L^{p}\left(d v_{\alpha}\right), 1<p \leqslant 2, q=\frac{p}{p-1}$ such that

$$
\left\|r^{b} f\right\|_{2 p, v_{\alpha}}+\|\psi\|_{2, v_{\alpha}}^{-\frac{2}{9}}\left\|r^{b} f\right\|_{2, v_{\alpha}}<+\infty .
$$

Denote by $\chi_{\Sigma}$ the characteristic function associated to $\Sigma$. Using Minkowski's inequality, relations (5) and (7), we obtain for every $\rho>0$

$$
\begin{aligned}
\left\|\chi_{\Sigma} S_{\psi}^{\alpha}(f)\right\|_{q, \mu_{\alpha}} & \leqslant\left\|\chi_{\Sigma} S_{\psi}^{\alpha}\left(\chi_{[0, \rho} f\right)\right\|_{q, \mu_{\alpha}}+\left\|\chi_{\Sigma} S_{\psi}^{\alpha}(f)\left(\chi_{[\rho,+\infty[} f\right)\right\|_{q, \mu_{\alpha}} \\
& \leqslant\left(\mu_{\alpha}(\Sigma)\right)^{\frac{1}{q}}\left\|S_{\psi}^{\alpha}\left(\chi_{[0, \rho[} f\right)\right\|_{\infty, \mu_{\alpha}}+\left\|S_{\psi}^{\alpha}(f)\left(\chi_{[\rho,+\infty[} f\right)\right\|_{q, \mu_{\alpha}} \\
& \leqslant\left(\mu_{\alpha}(\Sigma)\right)^{\frac{1}{q}}\|\psi\|_{2, v_{\alpha}}\left\|\chi_{[0, \rho[} f\right\|_{2, v_{\alpha}}+C_{\psi}^{\frac{1}{q}}\|\psi\|_{2, v_{\alpha}}^{1-\frac{2}{q}}\left\|\chi_{[\rho,+\infty[} f\right\|_{2, v_{\alpha}} .
\end{aligned}
$$

On the other hand, by Hölder's inequality

$$
\left\|\chi_{[0, \rho[} f\right\|_{2, v_{\alpha}} \leqslant\left\|r^{-b} \chi_{[0, \rho[}\right\|_{2 q, v_{\alpha}}\left\|r^{b} f\right\|_{2 p, v_{\alpha}} .
$$

By simple calculus and the hypothesis $0<b<\frac{\alpha+1}{q}$, we obtain

$$
\left\|\chi_{[0, \rho[} f\right\|_{2, v_{\alpha}} \leqslant C_{b, \alpha, q} \rho^{\frac{\alpha+1}{q}-b}\left\|r^{b} f\right\|_{2 p, v_{\alpha}}
$$

where $C_{b, \alpha, q}=\left(\frac{1}{2^{\alpha+1} \Gamma(\alpha+1)(\alpha+1-b q)}\right)^{\frac{1}{2 q}}$. Moreover,

$$
\left\|\chi_{[\rho,+\infty[} f\right\|_{2, v_{\alpha}} \leqslant \rho^{-b}\left\|r^{b} f\right\|_{2, v_{\alpha}} .
$$

From (8) and (9), we get

$$
\left\|\chi_{\Sigma} S_{\psi}^{\alpha}(f)\right\|_{q, \mu_{\alpha}} \leqslant C_{b, \alpha, q}\left(\mu_{\alpha}(\Sigma)\right)^{\frac{1}{q}}\|\psi\|_{2, v_{\alpha}} \rho^{\frac{\alpha+1}{q}-b}\left\|r^{b} f\right\|_{2 p, v_{\alpha}}+\rho^{-b} C_{\psi}^{\frac{1}{q}}\|\psi\|_{2, v_{\alpha}}^{1-\frac{2}{q}}\left\|r^{b} f\right\|_{2, v_{\alpha}} .
$$

We choose

$$
\rho=\left(C_{b, \alpha, q}\right)^{\frac{-q}{\alpha+1}}\left(\mu_{\alpha}(\Sigma)\right)^{\frac{-1}{\alpha+1}} C_{\psi}^{\frac{1}{\alpha+1}}
$$


hence, we obtain the first inequality.

(ii) It is clear that the second inequality holds if $\|f\|_{2 p, v_{\alpha}}$ or $\left\|r^{b} f\right\|_{2 p, v_{\alpha}}=+\infty$. Assume that

$$
\|f\|_{2 p, v_{\alpha}}+\left\|r^{b} f\right\|_{2 p, v_{\alpha}}<+\infty
$$

From hypothesis $b>\frac{\alpha+1}{q}$, we deduce that the function $r \longrightarrow\left(1+r^{2 b p}\right)^{\frac{-1}{p}}$ belongs to $L^{q}\left(d v_{\alpha}\right)$ and By Hölder's inequality, we have

$$
\begin{aligned}
\|f\|_{2, v_{\alpha}}^{2 p} & =\left(\int_{0}^{+\infty}\left(1+r^{2 b p}\right)^{\frac{-1}{p}}\left(1+r^{2 b p}\right)^{\frac{1}{p}}|f(r)|^{2} d v_{\alpha}(r)\right)^{p} \\
& \leqslant\left(\int_{0}^{+\infty} \frac{d v_{\alpha}(r)}{\left(1+r^{2 b p}\right)^{\frac{q}{p}}}\right)^{\frac{p}{q}}\left(\|f\|_{2 p, v_{\alpha}}^{2 p}+\left\|r^{b} f\right\|_{2 p, v_{\alpha}}^{2 p}\right) .
\end{aligned}
$$

However, with a standard computation, we obtain

$$
\left(\int_{0}^{+\infty} \frac{d v_{\alpha}(r)}{\left(1+r^{2 b p}\right)^{\frac{q}{p}}}\right)^{\frac{p}{q}}=\left(\frac{\Gamma\left(\frac{\alpha+1}{b p}\right) \Gamma\left(\frac{q b-(\alpha+1)}{b p}\right)}{b p 2^{\alpha+1} \Gamma(\alpha+1) \Gamma\left(\frac{q}{p}\right)}\right)^{\frac{p}{q}}=M_{b, \alpha, q}^{\frac{p}{q}}
$$

Replacing $f(r)$ by $f_{t}(r)=f(r t), t>0$ in the relation (10), we deduce that for all $t>0$

$$
\|f\|_{2, v_{\alpha}}^{2 p} \leqslant M_{b, \alpha, q}^{\frac{p}{q}}\left(t^{(2 \alpha+2)(p-1)}\|f\|_{2 p, v_{\alpha}}^{2 p}+t^{(2 \alpha+2)(p-1)-2 p b}\left\|r^{b} f\right\|_{2 p, v_{\alpha}}^{2 p}\right) .
$$

In particular for $t=\left(\frac{(2 b p-(2 \alpha+2)(p-1))\left\|r^{b} f\right\|_{2 p, v_{\alpha}}^{2 p}}{(2 \alpha+2)(p-1)\|f\|_{2 p, v_{\alpha}}^{2 p}}\right)^{\frac{1}{2 b p}}$, we obtain

$$
\|f\|_{2, v_{\alpha}} \leqslant M_{b, \alpha, q}^{\frac{1}{2 q}}\left(\frac{q b}{q b-(\alpha+1)}\right)^{\frac{1}{2 p}}\left(\frac{q b}{\alpha+1}-1\right)^{\frac{\alpha+1}{2 q b p}}\|f\|_{2 p, v_{\alpha}}^{1-\frac{\alpha+1}{q b}}\left\|r^{b} f\right\|_{2 p, v_{\alpha}}^{\frac{\alpha+1}{q b}}
$$

Moreover,

$$
\begin{aligned}
\left\|\chi_{\Sigma} S_{\psi}^{\alpha}(f)\right\|_{q, \mu_{\alpha}} & \leqslant\left(\mu_{\alpha}(\Sigma)\right)^{\frac{1}{q}}\left\|S_{\psi}^{\alpha}(f)\right\|_{\infty, \mu_{\alpha}} \\
& \leqslant\left(\mu_{\alpha}(\Sigma)\right)^{\frac{1}{q}}\|f\|_{2, v_{\alpha}}\|\psi\|_{2, v_{\alpha}} \\
& \leqslant M_{b, \alpha, q}^{\frac{1}{2 q}}\left(\frac{q b}{q b-(\alpha+1)}\right)^{\frac{1}{2 p}}\left(\frac{q b}{\alpha+1}-1\right)^{\frac{\alpha+1}{2 q b p}}\left(\mu_{\alpha}(\Sigma)\right)^{\frac{1}{q}}\|f\|_{2 p, v_{\alpha}}^{1-\frac{\alpha+1}{q b}}\left\|r^{b} f\right\|_{2 p, v_{\alpha}}^{\frac{\alpha+1}{q b}}\|\psi\|_{2, v_{\alpha}} .
\end{aligned}
$$

This completes the proof of the second inequality.

(iii) Let $s>0$, from the inequality

$$
\left(\frac{r}{S}\right)^{\frac{\alpha+1}{2 q}} \leqslant 1+\left(\frac{r}{S}\right)^{\frac{\alpha+1}{q}}
$$

it follows that

$$
\left\|r^{\frac{\alpha+1}{2 q}} f\right\|_{2 p, v_{\alpha}} \leqslant s^{\frac{\alpha+1}{2 q}}\|f\|_{2 p, v_{\alpha}}+s^{\frac{-(\alpha+1)}{2 q}}\left\|r^{\frac{\alpha+1}{q}} f\right\|_{2 p, v_{\alpha}} .
$$

In particular, by choosing $s=\left\|r^{\frac{\alpha+1}{q}} f\right\|_{2 p, v_{\alpha}}^{\frac{q}{\alpha+1}}\|f\|_{2 p, v_{\alpha}}^{\frac{-q}{\alpha+1}}$, we obtain

$$
\left\|r^{\frac{\alpha+1}{2 q}} f\right\|_{2 p, v_{\alpha}} \leqslant 2\|f\|_{2 p, v_{\alpha}}^{\frac{1}{2}}\left\|r^{\frac{\alpha+1}{q}} f\right\|_{2 p, v_{\alpha}}^{\frac{1}{2}}
$$

Similarly, we prove that

$$
\left\|r^{\frac{\alpha+1}{2 q}} f\right\|_{2, v_{\alpha}} \leqslant 2\|f\|_{2, v_{\alpha}}^{\frac{1}{2}}\left\|r^{\frac{\alpha+1}{q}} f\right\|_{2, v_{\alpha}}^{\frac{1}{2}}
$$


Thus, we deduce that

$$
\begin{aligned}
\left\|\chi_{\Sigma} S_{\psi}^{\alpha}(f)\right\|_{q, \mu_{\alpha}} & \leqslant C_{1}\left(\frac{\alpha+1}{2 q}, \psi\right)\left(\mu_{\alpha}(\Sigma)\right)^{\frac{1}{2 q}}\left(\left\|r^{\frac{\alpha+1}{2 q}} f\right\|_{2 p, v_{\alpha}}+\|\psi\|_{2, v_{\alpha}}^{-\frac{2}{q}}\left\|r^{\frac{\alpha+1}{2 q}} f\right\|_{2, v_{\alpha}}\right) \\
& \leqslant 2 C_{1}\left(\frac{\alpha+1}{2 q}, \psi\right)\left(\mu_{\alpha}(\Sigma)\right)^{\frac{1}{2 q}}\left(\|\psi\|_{2, v_{\alpha}}^{-\frac{2}{q}}\|f\|_{2, v_{\alpha}}^{\frac{1}{2}}\left\|r^{\frac{\alpha+1}{q}} f\right\|_{2, v_{\alpha}}^{\frac{1}{2}}+\|f\|_{2 p, v_{\alpha}}^{\frac{1}{2}}\left\|r^{\frac{\alpha+1}{q}} f\right\|_{2 p, v_{\alpha}}^{\frac{1}{2}}\right),
\end{aligned}
$$

which gives the result for $b=\frac{\alpha+1}{q}$.

From the $L^{p}$ local uncertainty principle, we can find the following $L^{p}$ Heisenberg-Pauli-Weyl uncertainty principle for the Hankel-Stockwell transform.

Theorem 3. [ $L^{p}$ Heisenberg-Pauli-Weyl uncertainty principle for the Hankel-Stockwell transform] Let $\psi$ be an admissible window function, $p \in] 1,2], q=\frac{p}{p-1}$, and $d>0$. Then for every $f \in L^{p}\left(d v_{\alpha}\right)$, we have

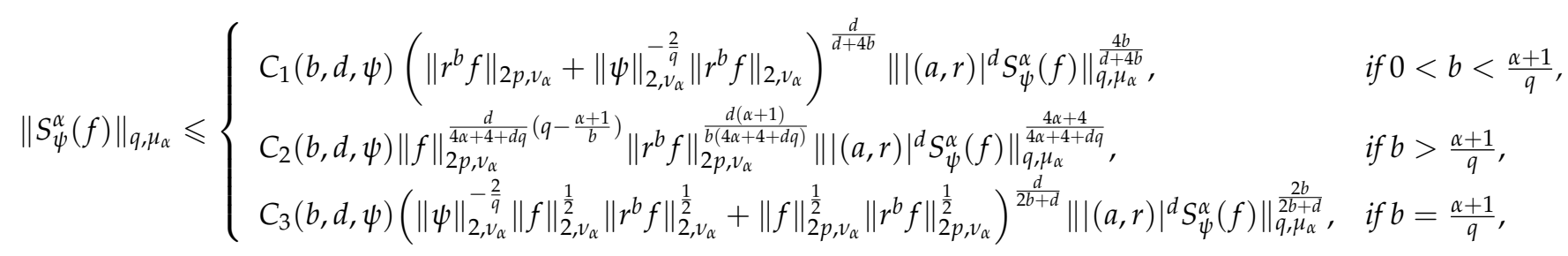

where

$$
\begin{aligned}
& C_{1}(b, d, \psi)=\frac{\left(C_{1}(b, \psi)\right)^{\frac{d}{d+4 b}}}{\left(2^{2 \alpha+2} \Gamma(2 \alpha+3)\right)^{\frac{d b}{(\alpha+1)(d+4 b)}}}\left(\left(\frac{d}{4 b}\right)^{\frac{4 b}{d+4 b}}+\left(\frac{4 b}{d}\right)^{\frac{d}{d+4 b}}\right)^{\frac{1}{q}}, \\
& C_{2}(b, d, \psi)=\frac{\left(C_{2}(b, \psi)\right)^{\frac{d q}{4 \alpha+4+d q}}}{\left(2^{2 \alpha+2} \Gamma(2 \alpha+3)\right)^{\frac{d}{\alpha \alpha+4+d q}}}\left(\left(\frac{d q}{4 \alpha+4}\right)^{\frac{4 \alpha+4}{4 \alpha+4+d q}}+\left(\frac{4 \alpha+4}{d q}\right)^{\frac{d q}{4 \alpha+4+d q}}\right)^{\frac{1}{q}}, \text { and } \\
& C_{3}(b, d, \psi)=\frac{\left(C_{3}(b, \psi)\right)^{\frac{d}{d+2 b}}}{\left(2^{2 \alpha+2} \Gamma(2 \alpha+3)\right)^{\frac{d}{2 q(d+2 b)}}}\left(\left(\frac{d}{2 b}\right)^{\frac{2 b}{d+2 b}}+\left(\frac{2 b}{d}\right)^{\frac{d}{d+2 b}}\right)^{\frac{1}{q}} .
\end{aligned}
$$

Proof. (i) Let $0<b<\frac{\alpha+1}{q}, d>0$. For $\rho>0$, let $\widetilde{B}_{\rho}=\left\{(a, r) \in \mathbb{R}_{+}^{*} \times \mathbb{R}_{+} ; a^{2}+r^{2} \leqslant \rho^{2}\right\}$. Then

$$
\left\|S_{\psi}^{\alpha}(f)\right\|_{q, \mu_{\alpha}}^{q}=\left\|\chi_{\widetilde{B}_{\rho}} S_{\psi}^{\alpha}(f)\right\|_{q, \mu_{\alpha}}^{q}+\left\|\chi_{\widetilde{B}_{\rho}^{c}} S_{\psi}^{\alpha}(f)\right\|_{q, \mu_{\alpha}}^{q} .
$$

From Theorem 2, we get

$$
\left\|\chi_{\widetilde{B}_{\rho}} S_{\psi}^{\alpha}(f)\right\|_{q, \mu_{\alpha}}^{q} \leqslant C_{1}^{q}(b, \psi)\left(\mu_{\alpha}\left(\widetilde{B}_{\rho}\right)\right)^{\frac{b q}{\alpha+1}}\left(\left\|r^{b} f\right\|_{2 p, v_{\alpha}}+\|\psi\|_{2, v_{\alpha}}^{-\frac{2}{q}}\left\|r^{b} f\right\|_{2, v_{\alpha}}\right)^{q} .
$$

On the other hand,

$$
\mu_{\alpha}\left(\widetilde{B}_{\rho}\right)=\frac{\rho^{4 \alpha+4}}{2^{2 \alpha+2} \Gamma(2 \alpha+3)} .
$$

Using the previous result, we obtain

$$
\left\|\chi_{\widetilde{B}_{\rho}} S_{\psi}^{\alpha}(f)\right\|_{q, \mu_{\alpha}}^{q} \leqslant C_{1}^{q}(b, \psi)\left(\frac{\rho^{4 \alpha+4}}{2^{2 \alpha+2} \Gamma(2 \alpha+3)}\right)^{\frac{b q}{\alpha+1}}\left(\left\|r^{b} f\right\|_{2 p, v_{\alpha}}+\|\psi\|_{2, v_{\alpha}}^{-\frac{2}{q}}\left\|r^{b} f\right\|_{2, v_{\alpha}}\right)^{q} .
$$

Moreover,

$$
\left\|\chi_{\widetilde{B}_{\rho}^{c}} S_{\psi}^{\alpha}(f)\right\|_{q, \mu_{\alpha}}^{q} \leqslant \rho^{-d q}\left\||(a, r)|^{d} S_{\psi}^{\alpha}(f)\right\|_{q, \mu_{\alpha}}^{q} .
$$


By Combining relations (11), (12) and (13), we get

$$
\left\|S_{\psi}^{\alpha}(f)\right\|_{q, \mu_{\alpha}}^{q} \leqslant C_{1}^{q}(b, \psi)\left(\frac{\rho^{4 \alpha+4}}{2^{2 \alpha+2} \Gamma(2 \alpha+3)}\right)^{\frac{b q}{\alpha+1}}\left(\left\|r^{b} f\right\|_{2 p, v_{\alpha}}+\|\psi\|_{2, \nu_{\alpha}}^{-\frac{2}{q}}\left\|r^{b} f\right\|_{2, v_{\alpha}}\right)^{q}+\rho^{-d q}\left\||(a, r)|^{d} S_{\psi}^{\alpha}(f)\right\|_{q, \mu_{\alpha}}^{q} .
$$

We choose

$$
\rho=\left(\frac{d\left(2^{2 \alpha+2} \Gamma(2 \alpha+3)\right)^{\frac{b q}{\alpha+1}}}{4 b C_{1}^{q}(b, \psi)}\right)^{\frac{1}{(d+4 b) q}}\left(\frac{\left\||(a, r)|^{d} S_{\psi}^{\alpha}(f)\right\|_{q, \mu_{\alpha}}}{\left\|r^{b} f\right\|_{2 p, v_{\alpha}}+\|\psi\|_{2, \nu_{\alpha}}^{-\frac{2}{q}}\left\|r^{b} f\right\|_{2, v_{\alpha}}}\right)^{\frac{1}{d+4 b}},
$$

hence, we obtain the first inequality.

(ii) Let $b>\frac{\alpha+1}{q}, d>0$ and let $\rho>0$. From Theorem 2, we obtain

$$
\begin{aligned}
\left\|\chi_{\widetilde{B}_{\rho}} S_{\psi}^{\alpha}(f)\right\|_{q, \mu_{\alpha}}^{q} & \leqslant C_{2}^{q}(b, \psi) \mu_{\alpha}\left(\widetilde{B}_{\rho}\right)\|f\|_{2 p, v_{\alpha}}^{q-\frac{\alpha+1}{b}}\left\|r^{b} f\right\|_{2 p, v_{\alpha}}^{\frac{\alpha+1}{b}} \\
& =C_{2}^{q}(b, \psi) \frac{\rho^{4 \alpha+4}}{2^{2 \alpha+2} \Gamma(2 \alpha+3)}\|f\|_{2 p, v_{\alpha}}^{q-\frac{\alpha+1}{b}}\left\|r^{b} f\right\|_{2 p, v_{\alpha}}^{\frac{\alpha+1}{b}} .
\end{aligned}
$$

Combining the relations (11), (13) and (14), we get

$$
\left\|S_{\psi}^{\alpha}(f)\right\|_{q, \mu_{\alpha}}^{q} \leqslant C_{2}^{q}(b, \psi) \frac{\rho^{4 \alpha+4}}{2^{2 \alpha+2} \Gamma(2 \alpha+3)}\|f\|_{2 p, v_{\alpha}}^{q-\frac{\alpha+1}{b}}\left\|r^{b} f\right\|_{2 p, v_{\alpha}}^{\frac{\alpha+1}{b}}+\rho^{-d q}\left\||(a, r)|^{d} S_{\psi}^{\alpha}(f)\right\|_{q, \mu_{\alpha}}^{q} .
$$

We choose

$$
\rho=\left(\frac{d q 2^{2 \alpha+2} \Gamma(2 \alpha+3)\left\||(a, r)|^{d} S_{\psi}^{\alpha}(f)\right\|_{q, \mu_{\alpha}}^{q}}{(4 \alpha+4) C_{2}^{q}(b, \psi)\|f\|_{2 p, v_{\alpha}}^{q-\frac{\alpha+1}{b}}\left\|r^{b} f\right\|_{2 p, v_{\alpha}}^{\frac{\alpha+1}{b}}}\right)^{\frac{1}{4 \alpha+4+d q}},
$$

hence, we obtain the second inequality.

(iii) Let $b=\frac{\alpha+1}{q}, d>0$ and let $\rho>0$. From Theorem 2, we get

$$
\begin{aligned}
\left\|\chi_{\widetilde{B}_{\rho}} S_{\psi}^{\alpha}(f)\right\|_{q, \mu_{\alpha}}^{q} & \leqslant C_{3}^{q}(b, \psi)\left(\mu_{\alpha}\left(\widetilde{B}_{\rho}\right)\right)^{\frac{1}{2}}\left(\|\psi\|_{2, v_{\alpha}}^{-\frac{2}{q}}\|f\|_{2, v_{\alpha}}^{\frac{1}{2}}\left\|r^{b} f\right\|_{2, v_{\alpha}}^{\frac{1}{2}}+\|f\|_{2 p, v_{\alpha}}^{\frac{1}{2}}\left\|r^{b} f\right\|_{2 p, v_{\alpha}}^{\frac{1}{2}}\right)^{q} \\
& =C_{3}^{q}(b, \psi) \frac{\rho^{2 \alpha+2}}{\sqrt{2^{2 \alpha+2} \Gamma(2 \alpha+3)}}\left(\|\psi\|_{2, v_{\alpha}}^{-\frac{2}{q}}\|f\|_{2, v_{\alpha}}^{\frac{1}{2}}\left\|r^{b} f\right\|_{2, v_{\alpha}}^{\frac{1}{2}}+\|f\|_{2 p, v_{\alpha}}^{\frac{1}{2}}\left\|r^{b} f\right\|_{2 p, v_{\alpha}}^{\frac{1}{2}}\right)^{q} .
\end{aligned}
$$

Combining the relations (11), (13) and (15), we obtain

$$
\begin{aligned}
\left\|S_{\psi}^{\alpha}(f)\right\|_{q, \mu_{\alpha}}^{q} \leqslant & C_{3}^{q}(b, \psi) \frac{\rho^{2 \alpha+2}}{\sqrt{2^{2 \alpha+2} \Gamma(2 \alpha+3)}}\left(\|\psi\|_{2, \nu_{\alpha}}^{-\frac{2}{q}}\|f\|_{2, v_{\alpha}}^{\frac{1}{2}}\left\|r^{b} f\right\|_{2, v_{\alpha}}^{\frac{1}{2}}+\|f\|_{2 p, v_{\alpha}}^{\frac{1}{2}}\left\|r^{b} f\right\|_{2 p, v_{\alpha}}^{\frac{1}{2}}\right)^{q} \\
& +\rho^{-d q}\left\||(a, r)|^{d} S_{\psi}^{\alpha}(f)\right\|_{q, \mu_{\alpha}}^{q} .
\end{aligned}
$$

We choose

$$
\rho=\left(\frac{d q\left(2^{2 \alpha+2} \Gamma(2 \alpha+3)\right)^{\frac{1}{2}}}{C_{3}^{q}(b, \psi)(2 \alpha+2)}\right)^{\frac{1}{2 \alpha+2+d q}} \times\left(\frac{\left\||(a, r)|^{d} S_{\psi}^{\alpha}(f)\right\|_{q, \mu_{\alpha}}}{\left(\|\psi\|_{2, v_{\alpha}}^{-\frac{2}{q}}\|f\|_{2, v_{\alpha}}^{\frac{1}{2}}\left\|r^{b} f\right\|_{2, v_{\alpha}}^{\frac{1}{2}}+\|f\|_{2 p, v_{\alpha}}^{\frac{1}{2}}\left\|r^{b} f\right\|_{2 p, v_{\alpha}}^{\frac{1}{2}}\right)}\right)^{\frac{q}{2 \alpha+2+d q}}
$$

hence, we obtain the result.

In the following, we shall use the $L^{p}$ Heisenberg-Pauli-Weyl uncertainty principle to obtain a concentration uncertainty principle. 
Definition 3. Let $0 \leqslant \varepsilon<1$ and let $S$ be a measurable set of $\mathbb{R}_{+}$. We say that $f \in L^{p}\left(d v_{\alpha}\right), p \in[1,2]$, is $\varepsilon$-concentrated on $S$ in $L^{p}\left(d v_{\alpha}\right)$-norm if there is a measurable function $h$ vanishing outside $S$ such that

$$
\|f-h\|_{p, v_{\alpha}} \leqslant \varepsilon\|f\|_{p, v_{\alpha}} .
$$

We introduce a projection operator $P_{S}$ as $P_{S} f(r)=f(r), \quad$ if $\quad r \in S$ and $P_{S} f(r)=0, \quad$ if $\quad r \notin S$. Let $0 \leqslant \varepsilon_{S}<1$. Then $f$ is $\varepsilon_{S}$-concentrated on $S$ in $L^{p}\left(d v_{\alpha}\right)$-norm if and only if

$$
\left\|f-P_{S} f\right\|_{p, v_{\alpha}} \leqslant \varepsilon_{S}\|f\|_{p, v_{\alpha}} .
$$

Definition 4. Let $\psi$ be an admissible window function and $\Sigma$ be a measurable set of $\mathbb{R}_{+}^{*} \times \mathbb{R}_{+}$. We define a projection operator $Q_{\Sigma}$ as

$$
Q_{\Sigma} f=\left(S_{\psi}^{\alpha}\right)^{-1}\left(P_{\Sigma}\left(S_{\psi}^{\alpha}(f)\right)\right)
$$

Let $0 \leqslant \varepsilon_{\Sigma}<1$. Then $S_{\psi}^{\alpha}$ is $\varepsilon_{\Sigma}$-concentrated on $\Sigma$ in $L^{q}\left(d \mu_{\alpha}\right)$-norm, $1 \leqslant q \leqslant 2$ if and only if

$$
\left\|S_{\psi}^{\alpha}(f)-S_{\psi}^{\alpha}\left(Q_{\Sigma} f\right)\right\|_{q, \mu_{\alpha}} \leqslant \varepsilon_{\Sigma}\left\|S_{\psi}^{\alpha}(f)\right\|_{q, \mu_{\alpha}} .
$$

Proposition 4. Let $\psi$ be an admissible window function and $\Sigma$ be a measurable set of $\mathbb{R}_{+}^{*} \times \mathbb{R}_{+}$. Then, for every $p>2$ and $\varepsilon>0$, if $S_{\psi}^{\alpha}$ is $\varepsilon$-concentrated in $\Sigma$ with respect to the norm $\|\cdot\|_{2, \mu_{\alpha}}$, then

$$
\left(\mu_{\alpha}(\Sigma)\right)^{\frac{p-2}{p}} \geqslant\left(1-\varepsilon^{2}\right) C_{\psi}^{1-\frac{2}{p}}\|\psi\|_{2, v_{\alpha}}^{\frac{4}{p}-2}
$$

where $\mu_{\alpha}(\Sigma)=\iint_{\Sigma} d v_{\alpha}(a) d v_{\alpha}(r)$.

Proof. Let $f \in L^{2}\left(d v_{\alpha}\right)$ and $p>2$. As $S_{\psi}^{\alpha}(f)$ is $\varepsilon$-concentrated in $\Sigma$ with respect to the norm $\|\cdot\|_{2, \mu_{\alpha}}$, we have

$$
\left\|\chi_{\Sigma^{c}} S_{\psi}^{\alpha}(f)\right\|_{2, \mu_{\alpha}} \leqslant \varepsilon \sqrt{C_{\psi}}\|f\|_{2, v_{\alpha}} .
$$

Now, using relation (6), we get

$$
\left\|\chi_{\Sigma} S_{\psi}^{\alpha}(f)\right\|_{2, \mu_{\alpha}}^{2} \geqslant\left(1-\varepsilon^{2}\right) C_{\psi}\|f\|_{2, v_{\alpha}}^{2} .
$$

Applying Hölder's inequality, we obtain

$$
\left\|\chi_{\Sigma} S_{\psi}^{\alpha}(f)\right\|_{2, \mu_{\alpha}}^{2} \leqslant\left\|S_{\psi}^{\alpha}(f)\right\|_{p, \mu_{\alpha}}^{2}\left(\mu_{\alpha}(\Sigma)\right)^{\frac{p-2}{p}} .
$$

By relation (7), we obtain

$$
\left\|\chi_{\Sigma} S_{\psi}^{\alpha}(f)\right\|_{2, \mu_{\alpha}}^{2} \leqslant C_{\psi}^{\frac{2}{p}}\|\psi\|_{2, \nu_{\alpha}}^{2-\frac{4}{p}}\|f\|_{2, v_{\alpha}}^{2}\left(\mu_{\alpha}(\Sigma)\right)^{\frac{p-2}{p}}
$$

Finally,

$$
\left(\mu_{\alpha}(\Sigma)\right)^{\frac{p-2}{p}} \geqslant\left(1-\varepsilon^{2}\right) C_{\psi}^{1-\frac{2}{p}}\|\psi\|_{2, \nu_{\alpha}}^{\frac{4}{p}-2}
$$

Proposition 5. Let $\psi$ be an admissible window function and $f \in L^{1}\left(d v_{\alpha}\right) \cap L^{2}\left(d v_{\alpha}\right)$ such that $\left\|S_{\psi}^{\alpha}(f)\right\|_{2, \mu_{\alpha}}=1$. If $f$ is $\varepsilon_{S}$-concentrated on $S$ in $L^{1}\left(d v_{\alpha}\right)$-norm and $S_{\psi}^{\alpha}(f)$ is $\varepsilon_{\Sigma}$-concentrated on $\Sigma$ in $L^{2}\left(d \mu_{\alpha}\right)$-norm, then $v_{\alpha}(S) \geqslant$ $C_{\psi}\left(1-\varepsilon_{S}\right)^{2}\|f\|_{1, v_{\alpha}}^{2}$ and $\mu_{\alpha}(\Sigma)\|f\|_{2, v_{\alpha}}^{2}\|\psi\|_{2, v_{\alpha}}^{2} \geqslant 1-\varepsilon_{\Sigma}^{2}$.

Proof. As $S_{\psi}^{\alpha}(f)$ is $\varepsilon_{\Sigma}$-concentrated on $\Sigma$ in $L^{2}\left(d \mu_{\alpha}\right)$-norm and by the orthogonality of the projection operator $P_{\Sigma}$, it follows that

$$
\left\|S_{\psi}^{\alpha}(f)\right\|_{2, \mu_{\alpha}}^{2}-\left\|S_{\psi}^{\alpha}(f)-P_{\Sigma}\left(S_{\psi}^{\alpha}(f)\right)\right\|_{2, \mu_{\alpha}}^{2}=\left\|P_{\Sigma}\left(S_{\psi}^{\alpha}(f)\right)\right\|_{2, \mu_{\alpha}}^{2} \geqslant 1-\varepsilon_{\Sigma}^{2}
$$


and thus

$$
1-\varepsilon_{\Sigma}^{2} \leqslant\left\|S_{\psi}^{\alpha}(f)\right\|_{\infty, \mu_{\alpha}}^{2} \mu_{\alpha}(\Sigma) \leqslant \mu_{\alpha}(\Sigma)\|f\|_{2, v_{\alpha}}^{2}\|\psi\|_{2, v_{\alpha}}^{2} .
$$

By the same way, $f$ is $\varepsilon_{\mathcal{S}}$-concentrated on $S$ in $L^{1}\left(d v_{\alpha}\right)$-norm, we obtain

$$
\left(1-\varepsilon_{S}\right)\|f\|_{1, v_{\alpha}} \leqslant \int_{S}|f(r)| d v_{\alpha}(r) .
$$

Now, by the Cauchy-Schwarz inequality and the fact that $\|f\|_{2, v_{\alpha}}=\frac{1}{\sqrt{C_{\psi}}}$, we get

$$
\left(1-\varepsilon_{S}\right)\|f\|_{1, v_{\alpha}} \leqslant \frac{v_{\alpha}^{\frac{1}{2}}(S)}{\sqrt{C_{\psi}}} .
$$

Definition 5. Let $\psi$ be an admissible window function and $\Sigma$ be a measurable set of $\mathbb{R}_{+}^{*} \times \mathbb{R}_{+}$. Let $d>0$, $f \in L^{p}\left(d v_{\alpha}\right), p \in[1,2]$ and $0 \leqslant \varepsilon_{\Sigma}<1$. We say that $|(a, r)|^{d} S_{\psi}^{\alpha}$ is $\varepsilon_{\Sigma}$-concentrated on $\Sigma$ in $L^{q}\left(d \mu_{\alpha}\right)$-norm, if and only if

$$
\left\||(a, r)|^{d} S_{\psi}^{\alpha}(f)-|(a, r)|^{d} S_{\psi}^{\alpha}\left(Q_{\Sigma} f\right)\right\|_{q, \mu_{\alpha}} \leqslant \varepsilon_{\Sigma}\left\||(a, r)|^{d} S_{\psi}^{\alpha}(f)\right\|_{q, \mu_{\alpha}} .
$$

Theorem 4. Let $\psi$ be an admissible window function and $\Sigma$ be a measurable set of $\mathbb{R}_{+}^{*} \times \mathbb{R}_{+}$. Let $f \in L^{p}\left(d v_{\alpha}\right), p \in$ ] $1,2], 0 \leqslant \varepsilon_{\Sigma}<1$ and $d>0$. If $|(a, r)|^{d} S_{\psi}^{\alpha}$ is $\varepsilon_{\Sigma}$-concentrated on $\Sigma$ in $L^{q}\left(d \mu_{\alpha}\right)$-norm, then

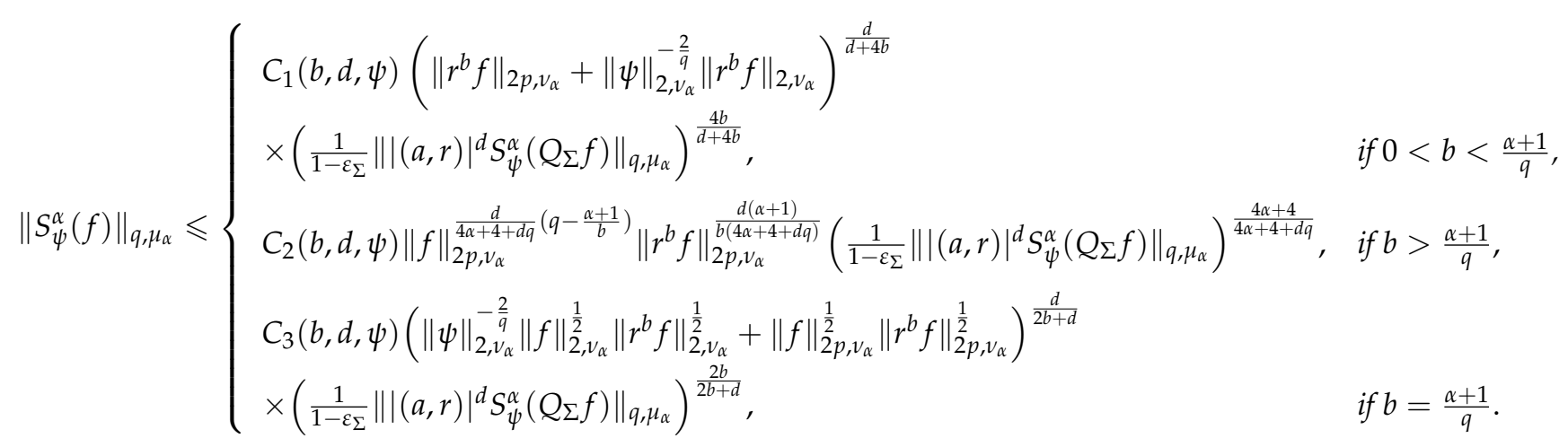

Proof. Let $\left.\left.f \in L^{p}\left(d v_{\alpha}\right), p \in\right] 1,2\right]$. Since $|(a, r)|^{d} S_{\psi}^{\alpha}$ is $\varepsilon_{\Sigma}$-concentrated on $\Sigma$ in $L^{q}\left(d \mu_{\alpha}\right)$-norm, then we have

$$
\left\||(a, r)|^{d} S_{\psi}^{\alpha}(f)\right\|_{q, \mu_{\alpha}} \leqslant\left\||(a, r)|^{d} S_{\psi}^{\alpha}\left(Q_{\Sigma} f\right)\right\|_{q, \mu_{\alpha}}+\varepsilon_{\Sigma}\left\||(a, r)|^{d} S_{\psi}^{\alpha}(f)\right\|_{q, \mu_{\alpha}} .
$$

Thus,

$$
\left\||(a, r)|^{d} S_{\psi}^{\alpha}(f)\right\|_{q, \mu_{\alpha}} \leqslant \frac{1}{1-\varepsilon_{\Sigma}}\left\||(a, r)|^{d} S_{\psi}^{\alpha}\left(Q_{\Sigma} f\right)\right\|_{q, \mu_{\alpha}},
$$

and we obtain the result from Theorem 3.

Definition 6. Let $\Sigma$ be a measurable set of $\mathbb{R}_{+}^{*} \times \mathbb{R}_{+}$and $0 \leqslant \eta<\sqrt{C_{\psi}}$. Then a nonzero function $f \in$ $L^{p}\left(d v_{\alpha}\right), 1 \leqslant p \leqslant 2$ is $\eta$-bandlimited on $\Sigma$ in $L^{q}\left(d \mu_{\alpha}\right)$-norm, if

$$
\left\|\chi_{\Sigma^{c}} S_{\psi}^{\alpha}(f)\right\|_{q, \mu_{\alpha}} \leqslant \eta\|f\|_{p, v_{\alpha}} .
$$

where $q=\frac{p}{p-1}$.

Corollary 1. Let $\psi$ be an admissible window function. 
(i) If $0<b<\frac{\alpha+1}{2}$, then there exists a positive constant $C$ such that for every function $f$ which is $\eta$-bandlimited on $\Sigma$

$$
\left(\mu_{\alpha}(\Sigma)\right)^{\frac{2 b}{\alpha+1}}\left(\left\|r^{b} f\right\|_{4, v_{\alpha}}+\|\psi\|_{2, v_{\alpha}}^{-1}\left\|r^{b} f\right\|_{2, v_{\alpha}}\right)^{2} \geqslant C\left(C_{\psi}-\eta^{2}\right)\|f\|_{2, v_{\alpha}}^{2} .
$$

(ii) If $b>\frac{\alpha+1}{2}$, then there exists a positive constant $C$ such that for every function $f$ which is $\eta$-bandlimited on $\Sigma$

$$
\mu_{\alpha}(\Sigma)\|f\|_{4, v_{\alpha}}^{2-\frac{\alpha+1}{b}}\left\|r^{b} f\right\|_{4, v_{\alpha}}^{\frac{\alpha+1}{b}} \geqslant C\left(C_{\psi}-\eta^{2}\right)\|f\|_{2, v_{\alpha}}^{2}
$$

Proof. Since $f \in L^{2}\left(d v_{\alpha}\right)$ is $\eta$-bandlimited on $\Sigma$, then

$$
\left\|\chi_{\Sigma} S_{\psi}^{\alpha}(f)\right\|_{2, \mu_{\alpha}}^{2}=C_{\psi}\|f\|_{2, \nu_{\alpha}}^{2}-\left\|\chi_{\Sigma^{c}} S_{\psi}^{\alpha}(f)\right\|_{2, \mu_{\alpha}}^{2} \geqslant\left(C_{\psi}-\eta^{2}\right)\|f\|_{2, \nu_{\alpha}}^{2} .
$$

For (i) and (ii), we use the local inequalities given respectively by Theorem 2.

According to the following Pitt's inequality for the Hankel transform [9], we obtain the Pitt's inequality for the Hankel-Stockwell transform.

Proposition 6. Let $0 \leqslant \eta<\alpha+1$. For every $f \in S_{e}(\mathbb{R})$, we have

$$
\int_{0}^{+\infty}|\lambda|^{-\eta}\left|\mathcal{H}_{\alpha}(f)(\lambda)\right|^{2} d v_{\alpha}(\lambda) \leqslant C_{\eta, \alpha} \int_{0}^{+\infty}|r|^{\eta}|f(r)|^{2} d v_{\alpha}(r),
$$

where $C_{\eta, \alpha}=2^{-\eta}\left(\frac{\Gamma\left(\frac{2 \alpha+2-\eta}{4}\right)}{\Gamma\left(\frac{2 \alpha+2+\eta}{4}\right)}\right)^{2}$ and $\Gamma($.$) denotes the well known Eurler's gamma function.$

Theorem 5. [Pitt's inequality the Hankel- Stockwell transform] Let $\psi$ be an admissible window function and $0 \leqslant \eta<\alpha+1$. For every $f \in S_{e}(\mathbb{R})$, the Pitt's inequality for the Hankel-Stockwell transform is given by

$$
C_{\psi} \int_{0}^{+\infty}|\lambda|^{-\eta}\left|\mathcal{H}_{\alpha}(f)(\lambda)\right|^{2} d v_{\alpha}(\lambda) \leqslant C_{\eta, \alpha} \int_{0}^{+\infty} \int_{0}^{+\infty}|r|^{\eta}\left|S_{\psi}^{\alpha}(f)(a, r)\right|^{2} d \mu_{\alpha}(a, r) .
$$

Proof. For $\eta=0$, the result follows from relation (6). Now suppose that $0<\eta<\alpha+1$. For every $f \in S_{e}(\mathbb{R})$ and by (16), we can write

$$
\int_{0}^{+\infty}|\lambda|^{-\eta}\left|\mathcal{H}_{\alpha}\left(S_{\psi}^{\alpha}(f)(a, .)\right)(\lambda)\right|^{2} d v_{\alpha}(\lambda) \leqslant C_{\eta, \alpha} \int_{0}^{+\infty}|r|^{\eta}\left|S_{\psi}^{\alpha}(f)(a, r)\right|^{2} d v_{\alpha}(r)
$$

Integrating with respect $d v_{\alpha}(a)$, we get

$$
C_{\eta, \alpha} \int_{0}^{+\infty} \int_{0}^{+\infty}|r|^{\eta}\left|S_{\psi}^{\alpha}(f)(a, r)\right|^{2} d \mu_{\alpha}(a, r) \geqslant \int_{0}^{+\infty} \int_{0}^{+\infty}|\lambda|^{-\eta}\left|\mathcal{H}_{\alpha}\left(S_{\psi}^{\alpha}(f)(a, .)\right)(\lambda)\right|^{2} d v_{\alpha}(a) d v_{\alpha}(\lambda) .
$$

By (1)-(4) and using Fubini's theorem, we obtain

$$
\begin{aligned}
\int_{0}^{+\infty} \int_{0}^{+\infty}|\lambda|^{-\eta}\left|\mathcal{H}_{\alpha}\left(S_{\psi}^{\alpha}(f)(a, .)\right)(\lambda)\right|^{2} d v_{\alpha}(a) d v_{\alpha}(\lambda) \\
=\int_{0}^{+\infty}|\lambda|^{-\eta}\left|\mathcal{H}_{\alpha}(f)(\lambda)\right|^{2}\left(\int_{0}^{+\infty}\left|\mathcal{H}_{\alpha}\left(D_{a} M_{1}(\psi)\right)(\lambda)\right|^{2} d v_{\alpha}(a)\right) d v_{\alpha}(\lambda) \\
=\int_{0}^{+\infty}|\lambda|^{-\eta}\left|\mathcal{H}_{\alpha}(f)(\lambda)\right|^{2}\left(\int_{0}^{+\infty}\left|D_{\frac{1}{a}}\left(\sqrt{\tau_{1}\left(\left|\mathcal{H}_{\alpha}(\psi)\right|^{2}\right)}\right)(\lambda)\right|^{2} d v_{\alpha}(a)\right) d v_{\alpha}(\lambda) \\
=\frac{1}{a^{\alpha+1}} \int_{0}^{+\infty}|\lambda|^{-\eta}\left|\mathcal{H}_{\alpha}(f)(\lambda)\right|^{2}\left(\int_{0}^{+\infty} D_{\frac{1}{a}}\left(\tau_{1}\left(\left|\mathcal{H}_{\alpha}(\psi)\right|^{2}\right)(\lambda)\right) d v_{\alpha}(a)\right) d v_{\alpha}(\lambda) \\
=\frac{1}{a^{2 \alpha+2}} \int_{0}^{+\infty}|\lambda|^{-\eta}\left|\mathcal{H}_{\alpha}(f)(\lambda)\right|^{2}\left(\int_{0}^{+\infty} \tau_{1}\left(\left|\mathcal{H}_{\alpha}(\psi)\right|^{2}\right)\left(\frac{\lambda}{a}\right) d v_{\alpha}(a)\right) d v_{\alpha}(\lambda) \\
=C_{\psi} \int_{0}^{+\infty}|\lambda|^{-\eta}\left|\mathcal{H}_{\alpha}(f)(\lambda)\right|^{2} d v_{\alpha}(\lambda)
\end{aligned}
$$


Relations (17) and (18) gives the Pitt's inequality for the Hankel-Stockwell transform.

Now, using the following logarithmic uncertainty principle for the Hankel transform [9], we obtain the logarithmic uncertainty principle for the Hankel-Stockwell transform.

Proposition 7. For every $f \in S_{e}(\mathbb{R})$, the following inequality holds:

$$
\int_{0}^{+\infty} \ln (t)\left|\mathcal{H}_{\alpha}(f)(t)\right|^{2} d v_{\alpha}(t)+\int_{0}^{+\infty} \ln (r)|f(r)|^{2} d v_{\alpha}(r) \geqslant\left(\ln 2+\omega\left(\frac{\alpha+1}{2}\right)\right) \int_{0}^{+\infty}|f(r)|^{2} d v_{\alpha}(r),
$$

where $\omega$ denotes the logarithmic derivative of the gamma function $\Gamma[20,21]$.

Theorem 6. [Logarithmic uncertainty principle for the Hankel-Stockwell transform] Let $\psi$ be an admissible window function. For every $f \in S_{e}(\mathbb{R})$, we have

$$
C_{\psi} \int_{0}^{+\infty} \ln (t)\left|\mathcal{H}_{\alpha}(f)(t)\right|^{2} d v_{\alpha}(t)+\int_{0}^{+\infty} \int_{0}^{+\infty} \ln (r)\left|S_{\psi}^{\alpha}(f)(a, r)\right|^{2} d \mu_{\alpha}(a, r) \geqslant C_{\psi}\left(\ln 2+\omega\left(\frac{\alpha+1}{2}\right)\right)\|f\|_{2, v_{\alpha}}^{2} .
$$

Proof. Replacing $f$ by $S_{\psi}^{\alpha}(f)$ in the inequality (19), we obtain

$$
\begin{aligned}
\int_{0}^{+\infty} \ln (t)\left|\mathcal{H}_{\alpha}\left(S_{\psi}^{\alpha}(f)(a, .)\right)(t)\right|^{2} d v_{\alpha}(t)+\int_{0}^{+\infty} \ln (r)\left|S_{\psi}^{\alpha}(f)(a, r)\right|^{2} d v_{\alpha}(r) \\
\geqslant\left(\ln 2+\omega\left(\frac{\alpha+1}{2}\right)\right) \int_{0}^{+\infty}\left|S_{\psi}^{\alpha}(f)(a, r)\right|^{2} d v_{\alpha}(r) .
\end{aligned}
$$

Integrating both sides with respect to $a$, we have

$$
\begin{aligned}
\int_{0}^{+\infty} \int_{0}^{+\infty} \ln (t)\left|\mathcal{H}_{\alpha}\left(S_{\psi}^{\alpha}(f)(a, .)\right)(t)\right|^{2} d \mu_{\alpha}(a, t)+\int_{0}^{+\infty} \int_{0}^{+\infty} \ln (r)\left|S_{\psi}^{\alpha}(f)(a, r)\right|^{2} d \mu_{\alpha}(a, r) \\
\geqslant\left(\ln 2+\omega\left(\frac{\alpha+1}{2}\right)\right) \int_{0}^{+\infty} \int_{0}^{+\infty}\left|S_{\psi}^{\alpha}(f)(a, r)\right|^{2} d \mu_{\alpha}(a, r) .
\end{aligned}
$$

By (1), (4) and using Fubini's theorem, we obtain

$$
\begin{aligned}
\int_{0}^{+\infty} \int_{0}^{+\infty} \ln (t)\left|\mathcal{H}_{\alpha}\left(S_{\psi}^{\alpha}(f)(a, .)\right)(t)\right|^{2} d \mu_{\alpha}(a, t) \\
=\int_{0}^{+\infty} \ln (t)\left|\mathcal{H}_{\alpha}(f)(t)\right|^{2}\left(\int_{0}^{+\infty}\left|\mathcal{H}_{\alpha}\left(D_{a} M_{1}(\psi)\right)(t)\right|^{2} d v_{\alpha}(a)\right) d v_{\alpha}(t) \\
=C_{\psi} \int_{0}^{+\infty} \ln (t)\left|\mathcal{H}_{\alpha}(f)(t)\right|^{2} d v_{\alpha}(t)
\end{aligned}
$$

Hence, by (6), (20) and (21), we have

$$
C_{\psi} \int_{0}^{+\infty} \ln (t)\left|\mathcal{H}_{\alpha}(f)(t)\right|^{2} d v_{\alpha}(t)+\int_{0}^{+\infty} \int_{0}^{+\infty} \ln (r)\left|S_{\psi}^{\alpha}(f)(a, r)\right|^{2} d \mu_{\alpha}(a, r) \geqslant C_{\psi}\left(\ln 2+\omega\left(\frac{\alpha+1}{2}\right)\right)\|f\|_{2, v_{\alpha}}^{2} .
$$

Conflicts of Interest: "The author declares no conflict of interest."

\section{References}

[1] Cowling, M. G., \& Price, J. F. (1984). Bandwidth versus time concentration: the Heisenberg-Pauli-Weyl inequality. SIAM Journal on Mathematical Analysis, 15(1), 151-165.

[2] Price, J. F. (1983). Inequalities and local uncertainty principles. Journal of Mathematical Physics, 24(7), $1711-1714$.

[3] Hleili, K. (2018). Uncertainty principles for spherical mean $L^{2}$-multiplier operators. Journal of Pseudo-Differential Operators and Applications, 9(3), 573-587.

[4] Hleili, K. (2020). Some results for the windowed Fourier transform related to the spherical mean operator. Acta Mathematica Vietnamica, 2020, 1-23. 
[5] Rösler, M. (1999). An uncertainty principle for the Dunkl transform. Bulletin of the Australian Mathematical Society, 59(3), 353-360.

[6] Banerjee, P. P., Nehmetallah, G., \& Chatterjee, M. R. (2005). Numerical modeling of cylindrically symmetric nonlinear self-focusing using an adaptive fast Hankel split-step method. Optics communications, 249(1-3), 293-300.

[7] Lohmann, A. W., Mendlovic, D., Zalevsky, Z., \& Dorsch, R. G. (1996). Some important fractional transformations for signal processing. Optics Communications, 125(1-3), 18-20.

[8] Bowie, P. C. (1971). Uncertainty inequalities for Hankel transforms. SIAM Journal on Mathematical Analysis, 2(4), 601-606.

[9] Omri, S. (2011). Logarithmic uncertainty principle for the Hankel transform. Integral Transforms and Special Functions, 22(9), 655-670.

[10] Tuan, V. K. (2007). Uncertainty principles for the Hankel transform. Integral Transforms and Special Functions, 18(5), 369-381.

[11] Hleili, K. (2018). Continuous wavelet transform and uncertainty principle related to the Weinstein operator. Integral Transforms and Special Functions, 29(4), 252-268.

[12] Hleili, K.(2020). A dispersion inequality and accumulated spectrograms in the weinstein setting. Bulletin of Mathematical Analysis and Applications, 12(1), 51-70.

[13] Ghobber, S. (2015). Shapiro's uncertainty principle in the Dunkl setting. Bulletin of the Australian Mathematical Society, 92(1), 98-110.

[14] Hamadi, N. B., Hafirassou, Z., \& Herch, H. (2020). Uncertainty principles for the Hankel-Stockwell transform. Journal of Pseudo-Differential Operators and Applications, 11(2), 543-564.

[15] Faris, W. G. (1978). Inequalities and uncertainty principles. Journal of Mathematical Physics, 19(2), 461-466.

[16] Price, J. F. (1987). Sharp local uncertainty inequalities. Studia Mathematica, 85, 37-45.

[17] Donoho, D. L., \& Stark, P. B. (1989). Uncertainty principles and signal recovery. SIAM Journal on Applied Mathematics, 49(3), 906-931.

[18] Soltani, F. (2014). $L^{p}$ local uncertainty inequality for the Sturm-Liouville transform. Cubo (Temuco), 16(1), 95-104.

[19] Schwartz, A. L. (1969). An inversion theorem for Hankel transforms. Proceedings of the American Mathematical Society, 22(3), 713-717.

[20] Andrews, G. E., Askey, R., \& Roy, R. (1999). Special Functions (No. 71). Cambridge university press.

[21] Silverman, R. A. (1972). Special Functions and their Applications. Dover Publications.

(C) 2021 by the authors; licensee PSRP, Lahore, Pakistan. This article is an open access article distributed under the terms and conditions of the Creative Commons Attribution (CC-BY) license (http://creativecommons.org/licenses/by/4.0/). 